\title{
Impact of Employer-Sponsored Onsite Pharmacy and Condition Management Programs on Medication Adherence
}

\author{
Kathleen M. Aguilar, MPH; Qingjiang Hou, MS; and Ross M. Miller, MD, MPH
}

\begin{abstract}
BACKGROUND: Poor medication adherence is associated with worsened health outcomes and higher health care expenditures. An increasing number of employers are sponsoring wellness initiatives designed to support healthy lifestyles, improve productivity, and offer a return on investment. Onsite pharmacies may facilitate higher medication adherence rates by providing employees a convenient, low-cost option for filling prescriptions that is integrated with other onsite health services.
\end{abstract}

OBJECTIVES: To (a) assess the impact of an employer's onsite pharmacy on health plan members' medication adherence using multiple measures of medication adherence and persistence, including medication possession ratio (MPR), average number of days until discontinuation (60-day gap in coverage), and percentage of members without a 30-day gap in coverage, and (b) evaluate these outcomes between those members who participated in condition management programs and those who did not.

METHODS: A retrospective analysis of a self-insured employer's claims data was undertaken. Medication adherence was assessed among the selfinsured employer's health plan members, which included subscribers and their dependents who filled an asthma, depression, diabetes, hypertension, or hyperlipidemia medication at an onsite pharmacy, compared with those who used a community pharmacy. Multiple standard measures of medication adherence were considered. These measures included MPR, which was assessed for 1- and 2-year time periods. MPR was chosen because it is one of the most commonly referenced formulas in the literature and represents adherence over a fixed period of time. In addition, medication persistence was estimated by 30-day gaps in coverage and discontinuation of treatment. To assess the impact of onsite pharmacy use and account for covariate effects, the linear mixed model approach was applied with the logit transformed MPR as the response variable. An analysis of MPR among condition management participants was also performed.

RESULTS: In total, 2,498 subscribers and their dependents were included in the analysis. The average MPR at $\mathbf{3 6 5}$ days was significantly higher $(P<0.0001)$ among onsite pharmacy users for all medication types, ranging from $13 \%$ higher for depression medications to $20 \%$ higher for hypertension medications. This trend persisted at 730 days $(P<0.001)$, with average MPRs ranging from $6 \%$ higher for hyperlipidemia medications to $11 \%$ higher for hypertension medications. A mixed model analysis indicated that members who used the onsite pharmacy were 3.44 times more likely to demonstrate medication adherence $(95 \% \mathrm{Cl}=2.84-4.16 ; P<0.0001)$ at 365 days. Likewise, at 180 and 365 days, onsite pharmacy users were less likely to have 30 -day gaps in treatment. The average number of days until discontinuation (defined as a 60-day gap) was also significantly longer $(P<0.0001)$ among onsite pharmacy users, ranging from an average of 56 additional days for depression medications to 105 additional days for hypertension medications. While the average MPR tended to be higher among those subscribers and their dependents who participated in condition management programs, this trend was not statistically significant for all medication types.
CONCLUSIONS: Based on multiple measures, onsite pharmacy use was associated with higher medication adherence, while the results were inconclusive for condition management participation.

J Manag Care Spec Pharm. 2015;21(8):670-77

Copyright $\odot 2015$, Academy of Managed Care Pharmacy. All rights reserved.

\section{What is already known about this subject}

Lack of medication adherence can exacerbate medical conditions, resulting in worsened outcomes, increased use of health care services, and higher overall costs.

Employer-sponsored wellness initiatives aim to support healthy lifestyles while increasing productivity and containing health care expenditures among covered populations.

Onsite health services, including pharmacies and condition management programs, may improve medication adherence and reduce expenses by providing convenient, low-cost care that is integrated with other employer-sponsored initiatives.

\section{What this study adds}

This retrospective claim analysis evaluates the impact of onsite pharmacies and condition management programs with several different measures of medication adherence, including formulas that have been reported in similar studies and others that are novel.

Based on multiple measures of medication adherence, onsite pharmacy use was associated with favorable adherence and persistence to treatment.

Similar trends were noted among condition management participants based on the least square regression results, although the mixed model analysis indicated a negative correlation between medication adherence and participation in condition management programs.

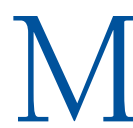
edication adherence refers to the extent to which patients take medications according to the prescribed dosing, timing, frequency, and duration. ${ }^{1}$ Failure to maintain medication adherence can exacerbate health conditions, which may result in worsened outcomes and an increased risk of mortality. ${ }^{2}$ Additionally, suboptimal medication adherence is associated with higher utilization of health care services and overall costs. ${ }^{3,4}$ 
Poor medication adherence is a considerable problem. Based on a 2004 meta-analysis of published studies, approximately $25 \%$ of patients do not take medications as prescribed. ${ }^{5}$ While the prevalence of nonadherence varies depending on the type of medication and study methodology, more recent studies have indicated higher rates, with suboptimal medication adherence frequently being observed in more than $50 \%$ of the patient populations. ${ }^{6-11}$ In the United States, excess costs due to this nonadherence are estimated to range from approximately $\$ 100$ to $\$ 300$ billion annually. ${ }^{12,13}$

An increasing number of employers are offering wellness programs and onsite health care services to improve the health of their workforce while containing rising health care expenses. ${ }^{14}$ By offering quality health care services focused on prevention and chronic condition management, often at the worksite, many organizations, such as Johnson \& Johnson and Highmark, Inc., have reduced costly expenses such as emergency room visits and inpatient stays. ${ }^{15-18}$ In 1 study, an employer-sponsored cardiac rehabilitation program resulted in a return on investment of $\$ 6$ for every dollar spent. ${ }^{19}$ Moreover, other reported benefits of onsite health care include improved productivity, staff morale, and health self-efficacy beliefs. ${ }^{20,21}$

Employer-hosted pharmacies and stand-alone dispensaries can be integrated into onsite wellness programs. Like other onsite wellness services, these pharmacies may offer employers a return on investment by helping to reduce other types of health care utilization and expenses. In particular, onsite pharmacies may have certain advantages that increase medication adherence. Such benefits often include the convenience of filling prescriptions at the workplace, reduced copays, and integration with other onsite health services. ${ }^{22-26}$

The primary objective of this study was to assess the impact of an employer's onsite pharmacy on health plan members' medication adherence using multiple measures of medication adherence and persistence, including calculations of medication possession ratio (MPR), average number of days until discontinuation (60-day gap in coverage), and percentage of members without a 30-day gap in coverage. As a secondary objective, these outcomes were also evaluated among those members who participated in condition management programs and those who did not.

\section{Methods}

\section{Study Design}

A retrospective claims analysis was performed to compare medication adherence rates among health plan members who used onsite wellness services. Cerner established the Healthe Clinic in 2006 to provide worksite health care to approximately 7,500 Cerner employees and their dependents in the Kansas City, Missouri, area. In addition to providing primary care and condition management services, the Healthe Clinic also offers an onsite pharmacy.
This onsite pharmacy has a number of features that may facilitate improved medication adherence among members who use it. In addition to the convenience of being located at the worksite, members may be more likely to fill their prescriptions at the onsite pharmacy due to discounted medication prices, automatic refills, and refill reminders for those who failed to pick-up a prescription. Similarly, pharmacists at the onsite pharmacy encourage members to fill 90-day medication supply whenever possible. The onsite pharmacy also provides free medication pill boxes as an adherence aid.

The effect of condition management program participation on medication adherence was also explored as a secondary objective. To aid members with chronic conditions, Cerner offers condition management programs that provide advisory sessions, educational materials, and other wellness tools. Subscribers and their dependents may participate in the condition management programs, although most of those who use these services are Cerner employees (either subscribers or dependents who are also employed by Cerner). These condition management programs are focused on hypertension, diabetes, weight management, and depression. A multidisciplinary team composed of registered nurses, certified athletic trainers, and other professionals with backgrounds in nutrition, fitness, and wellness are responsible for coordinating the programs. In general, members who engage in a condition management program have monthly visits with a health coach, although interactions can happen more or less frequently depending on need.

Medication adherence was evaluated among members who participated in any program, regardless of prescription type, given the likelihood of chronic comorbidities in the study population and the potential that participation may have a positive effect on overall wellness. Results were not stratified by condition because the focus of the condition management programs was not directly aligned with medication types included in this study (i.e., asthma, depression, diabetes, hyperlipidemia, and hypertension)

Cerner is a self-insured employer, and all health care claims are processed through its benefit administration, Cerner HealthPlan Services. Prescription data, demographic information, and condition management participation status were obtained from this claims database and de-identified to create the research dataset. An institutional review board (Western Institutional Review Board [WIRB]) determined that the protocol for this study qualified for an exception because the analysis was performed on a de-identified secondary dataset. WIRB is registered with the Office of Human Research Protections/ Food and Drug Administration, has been fully accredited by the Association for the Accreditation of Human Research Protection Programs, and has electronic signature compliance with 21 CFR Part 11. 
FIGURE 1 Study Population by Medication Type

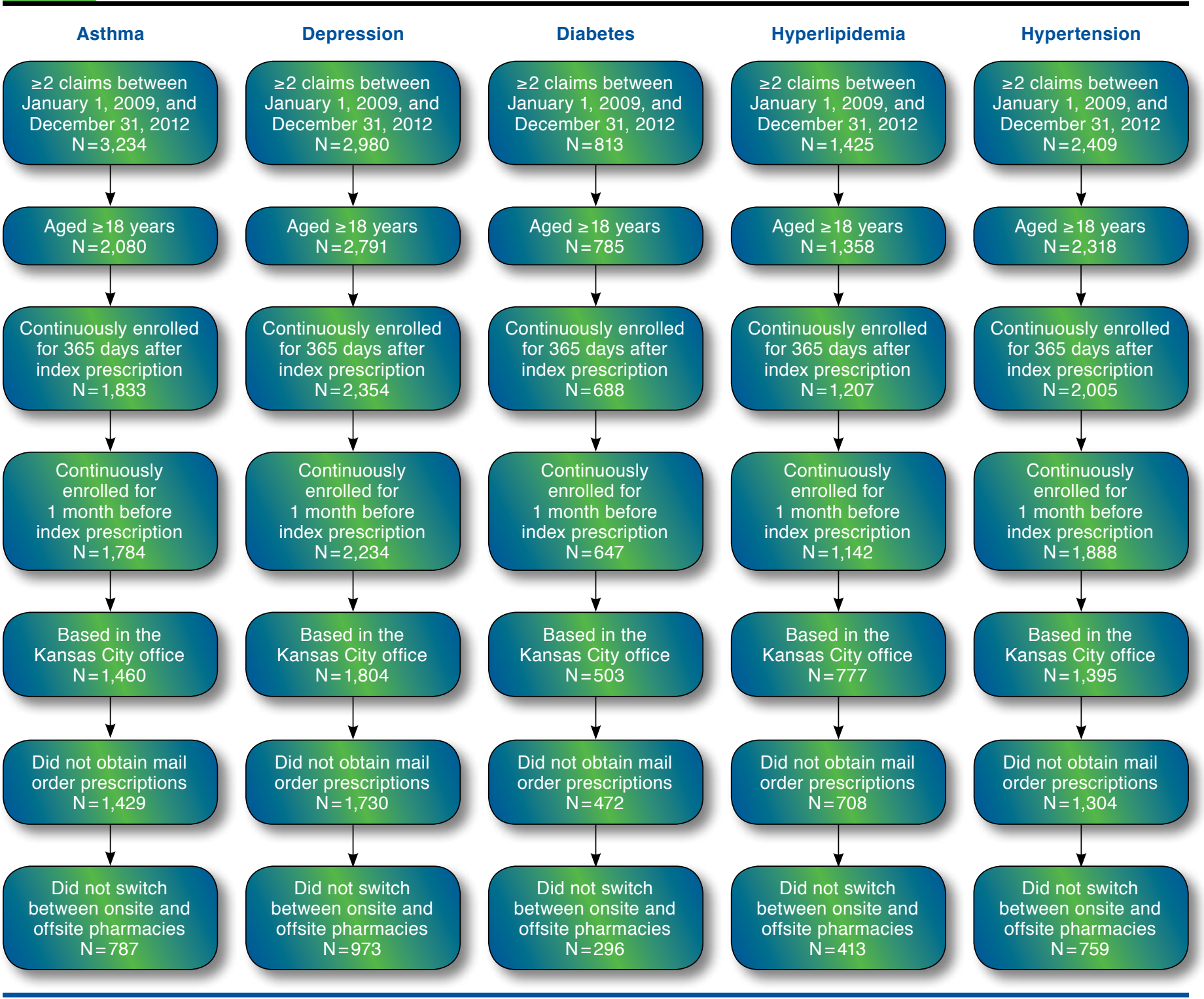

\section{Study Sample}

To be included in the analysis, members had to have a pharmacy claim for an asthma, depression, diabetes, hyperlipidemia, or hypertension medication during the intake period (January 1, 2009, to December 31, 2011) and a refill of the same medication category within 365 days. Additionally, members were required to have continuous health plan enrollment 1 month prior to and 365 days following the first prescription fill (i.e., the index prescription). The pre-index enrollment requirement was used to confirm comorbidities and determine whether members were new medication users (defined as those who had not filled a prescription for the drug class within the preceding 6 months). Those who were younger than 18 years, had a diagnosis of gestational diabetes, used mail-order prescription services, were based in an office outside of the Kansas City area, or switched between onsite and offsite pharmacies were excluded. A breakdown of the study population by medication type is presented in Figure 1.

\section{Study Variables}

To fully characterize medication adherence rates across onsite wellness users, the data were assessed through multiple standard formulas. First, MPR was calculated by dividing each member's total days of medication supply over 365-day 
TABLE 1 Characteristics of the Study Population

\begin{tabular}{|c|c|c|c|c|}
\hline \multirow[b]{2}{*}{ Age, mean [SD] } & \multicolumn{2}{|c|}{$\begin{array}{l}\text { Onsite Pharmacy } \\
\text { Users }(\mathrm{N}=1,305)\end{array}$} & \multicolumn{2}{|c|}{$\begin{array}{l}\text { Offsite Pharmacy } \\
\text { Users }(\mathrm{N}=1,193)\end{array}$} \\
\hline & 38.21 & {$[ \pm 11.3]$} & 38.15 & {$[ \pm 11.1]$} \\
\hline \multicolumn{5}{|l|}{ Gender, n (\%) } \\
\hline Male & 633 & $(48.5)$ & 451 & $(37.8)$ \\
\hline Female & 672 & $(51.5)$ & 742 & $(62.2)$ \\
\hline Onsite clinic users, n (\%)a & 1,072 & $(82.2)$ & 455 & $(17.6)$ \\
\hline \multicolumn{5}{|l|}{ Relationship status, n (\%) } \\
\hline Subscriber & 1,014 & $(77.7)$ & 636 & $(53.3)$ \\
\hline Dependent & 291 & $(22.3)$ & 557 & $(46.7)$ \\
\hline Mean CCI [SD] & 0.32 & {$[ \pm 0.68]$} & 0.4 & $\pm[0.94]$ \\
\hline \multicolumn{5}{|c|}{ Chronic condition medication use, n (\%) } \\
\hline Asthma & 305 & $(23.4)$ & 482 & $(40.4)$ \\
\hline Depression & 526 & $(40.3)$ & 447 & $(37.5)$ \\
\hline Diabetes & 177 & $(13.6)$ & 119 & $(10.0)$ \\
\hline Hyperlipidemia & 251 & $(19.2)$ & 162 & $(13.6)$ \\
\hline Hypertension & 437 & $(33.5)$ & 322 & $(27.0)$ \\
\hline $\begin{array}{l}\text { Condition management } \\
\text { program participation, n (\%) }\end{array}$ & 136 & (10.4) & 41 & $(3.4)$ \\
\hline \multicolumn{5}{|c|}{$\begin{array}{l}\text { a Members with } 3 \text { or more visits to the onsite clinic during the study period were } \\
\text { assumed to be onsite clinic users. } \\
C C I=\text { Charlson Comorbidity Index; SD=standard deviation. }\end{array}$} \\
\hline
\end{tabular}

and 730 -day periods. This formula was chosen because it is frequently referenced in the literature and represents adherence over a fixed period of time. MPR can be greater than 1 if members consistently refill prescriptions early; therefore, MPR results greater than 1 and less than 1.5 were truncated to 1 . MPRs that were greater than 1.5 may indicate underlying data concerns and were excluded. Note that 90-day refills accounted for approximately $16 \%$ of fills included in this study.

The individual MPRs were then averaged to determine the overall MPR for each medication category for the 2 time periods, stratified by pharmacy location or condition management participation. Members who received multiple medications within the same therapeutic drug class were assumed to have medication coverage each day that any drug in the therapeutic class was available. Consequently, the MPR estimate represents an upper bound of the actual adherence with prescribed therapy.

Next, medication persistence was evaluated by assessing gaps in coverage and discontinuation of treatment. Medication gaps were considered to be 30 days without a prescription refill, irrespective of previous early refills. For each medication type, the percentage of members without a 30-day gap in coverage at 180 and 365 days was calculated. A 60-day gap in coverage was assumed to be discontinuation of treatment, and the average number of days until discontinuation (i.e., the first day of the 60-day gap) was determined for each medication category.

In addition to medication adherence measures, characteristics of the study population were determined. These variables included demographic features, such as mean age, as well as comorbidities and use of the onsite clinic. ${ }^{27}$ A modified version of the Charlson Comorbidity Index (CCI) was used to calculate this information.

\section{Statistical Analysis}

Descriptive statistics for continuous variables (mean, median, and standard deviation) for each study group and medical condition were generated as a measure of central tendency and cross-group variation. For categorical variables, cross-tabulations in count along with group percentage were calculated. A chi-square test for count and the Student's t-test (cross-checked with the nonparametric Wilcoxon rank sum test) for continuous measure were performed to contrast onsite versus offsite differences for each of the 5 medical conditions. To identify independent factors associated with medication adherence, ordinary least square regression analyses on logit transformed MPR were performed for each medical condition. ${ }^{28}$ Linear regression with the mixed model methodology was utilized to account for dependency in patients with multiple medical conditions and to assess the overall effect of the onsite pharmacy on medication adherence, while adjusting for significant covariate effects. All statistical analysis was conducted with SAS 9.3 (SAS Institute, Inc., Carey, NC).

\section{Results}

In total, 2,498 members met inclusion criteria and were included in the analysis. Of these, 787 filled prescriptions for asthma, 973 for depression, 296 for diabetes, 413 for hyperlipidemia, and 759 for hypertension.

The demographic characteristics of the study population are presented in Table 1. Members who used the onsite pharmacy were more likely to also use the onsite health center and be the primary subscriber to the health plan. Offsite pharmacy users were more likely to be female and have a greater number of comorbid chronic conditions. Each medication type was well represented in the study, with at least $10 \%$ of each group receiving each of the medications.

Table 2 displays the medication adherence results by drug type. Across medication types, the average MPR at a fixed interval of 365 days for onsite pharmacy users was significantly higher $(P<0.0001$ for all medication types) than among offsite pharmacy users. Specifically, onsite pharmacy use was associated with MPRs that were greater for each medication type: asthma (14\% higher), depression (13\% higher), diabetes (16\% higher), hyperlipidemia (15\% higher), and hypertension (20\% higher). This trend persisted for the fixed 730-day interval $(P<0.001$ for all medication types): asthma ( $7 \%$ higher), depression ( $7 \%$ higher), diabetes ( $9 \%$ higher), hyperlipidemia (6\% higher), and hypertension (11\% higher).

For all medication types, the average days to discontinuation was also significantly higher $(P<0.0001$ for all medication types) among onsite users, ranging from an average of 56 


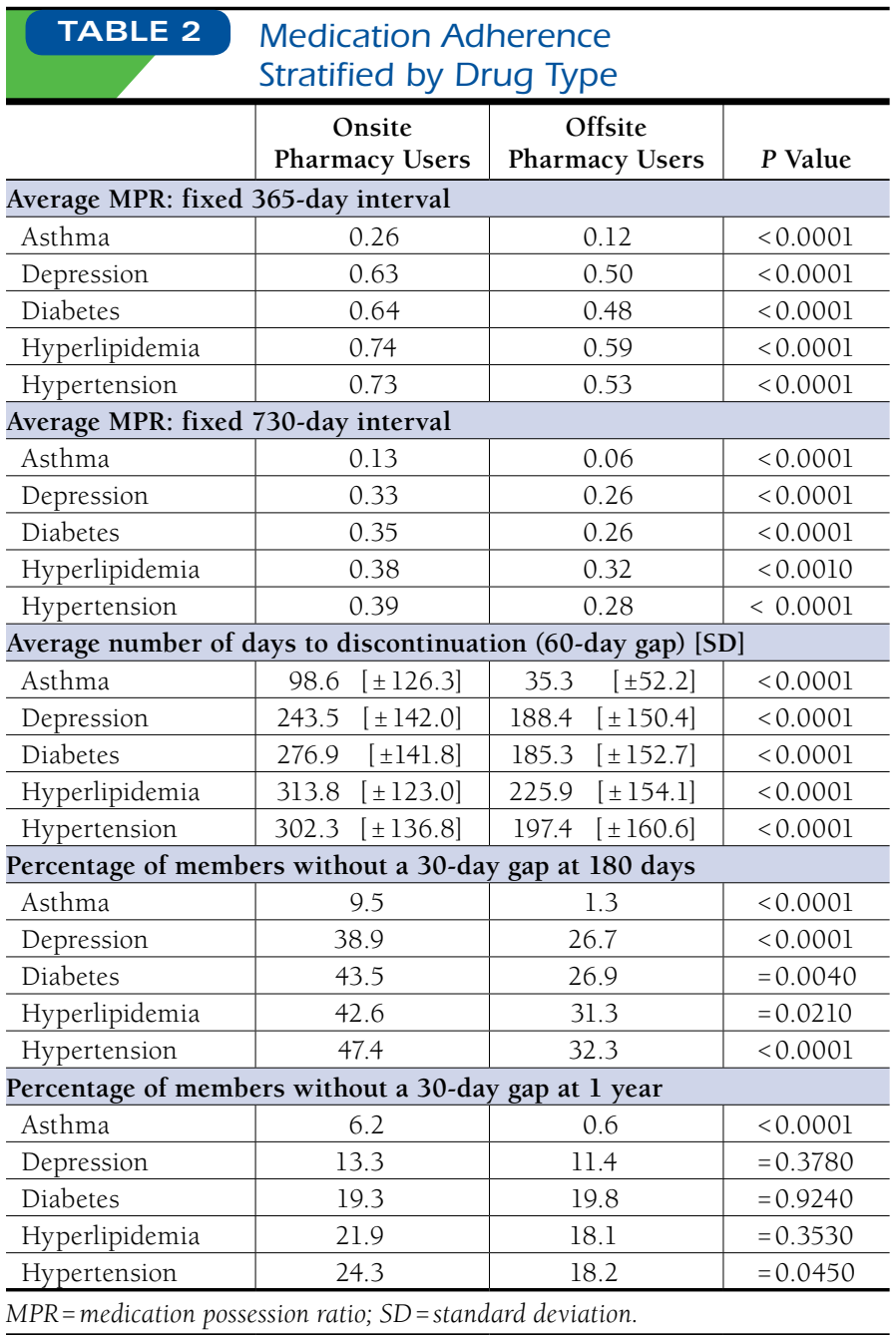

additional days for depression medications to 105 additional days for hypertension medications. Likewise, the percentage of members without a 30-day gap was significantly higher $(P<0.05$ for all medication types) across medication types at 180 days and trended higher at 1 year, although not all differences reached significance.

A mixed model analysis combining all 5 medical conditions indicated that, compared with offsite pharmacy users, the covariate adjusted odds of increasing medication adherence for onsite pharmacy user was significantly higher (odds ratio $=3.44 ; 95 \%$ confidence interval $[\mathrm{CI}]=2.84-4.16$; $P<0.0001)$. Moreover, these mixed model analyses also suggest that medication adherence is positively associated with age and CCI score. In contrast, a negative association with condition management participation was also observed (Table 3).

Using the mixed model approach, no significant differences in medication adherence between subscribers and dependents were observed. Based on an unadjusted subset analysis, no
TABLE 3 Average MPR Fixed 365-Day Interval Mixed Model Analyses

\begin{tabular}{l|c|c|c|c}
\hline & $\begin{array}{c}\text { Parameter } \\
\text { Estimate }\end{array}$ & SE & $\begin{array}{c}\text { Adjusted } \\
\text { OR }\end{array}$ & 95\% CI \\
\hline Onsite pharmacy use & 1.235 & 0.097 & 3.44 & $2.84-4.16$ \\
\hline Member age & 0.043 & 0.004 & 1.04 & $1.03-1.05$ \\
\hline CCI score & 0.353 & 0.061 & 1.42 & $1.26-1.61$ \\
\hline $\begin{array}{l}\text { Condition management } \\
\text { participation }\end{array}$ & -0.409 & 0.167 & 0.66 & $0.48-0.92$ \\
\hline Subscriber (versus dependent) & 0.197 & 0.104 & 0.99 & $0.99-1.49$ \\
\hline
\end{tabular}

$\mathrm{CCI}=$ Charlson Comorbidity Index; $\mathrm{CI}=$ confidence interval; $\mathrm{MPR}=$ medication possession ratio; $O R=$ odds ratio; $S E=$ standard error.

significant difference in 365-day MPR was found between subscribers and dependents for onsite $(P=0.468)$ and offsite $(P=0.212)$ pharmacy users. After adjusting for onsite pharmacy use, age, CCI, and condition management participation, medication adherence was slightly higher for subscribers, but the difference did not reach statistical significance at the 5\% level $(P=0.068)$.

The average MPRs at 365 and 730 days among condition management participants and nonparticipants are presented in Figure 2. Although the findings varied by medication type, these least square regression analyses tended to demonstrate a positive association between condition management participation and medication adherence. The influence of condition management participation was highest among members who filled hyperlipidemia and hypertension medications at 365 and 730 days. Note, these results were not stratified by condition because the condition management program types were not aligned with the medication classes included in this study.

\section{Discussion}

The results of this study indicate that onsite pharmacy use is associated with higher medication adherence rates among the health plan members included in this analysis. This finding expands upon previous research by replicating similar findings, introducing other measures, and evaluating the trends over a longer time period. In particular, rather than using a single outcome measure, our analysis considered multiple medication adherence formulas, which was beneficial for characterizing multiple dimensions of adherence in the population and cross-validating the results.

Medication adherence among patients who used onsite pharmacies and clinics compared with those who used community-based facilities was evaluated by Sherman et al. (2009) using MPR over a 1-year period. ${ }^{29}$ The authors observed that MPR was approximately $10 \%$ higher for onsite pharmacy users. In our analysis, the average MPR among onsite pharmacy users was $13 \%$ to $20 \%$ higher $(P<0.0001)$ across included conditions at 1 year for onsite pharmacy users. Moreover, our research 


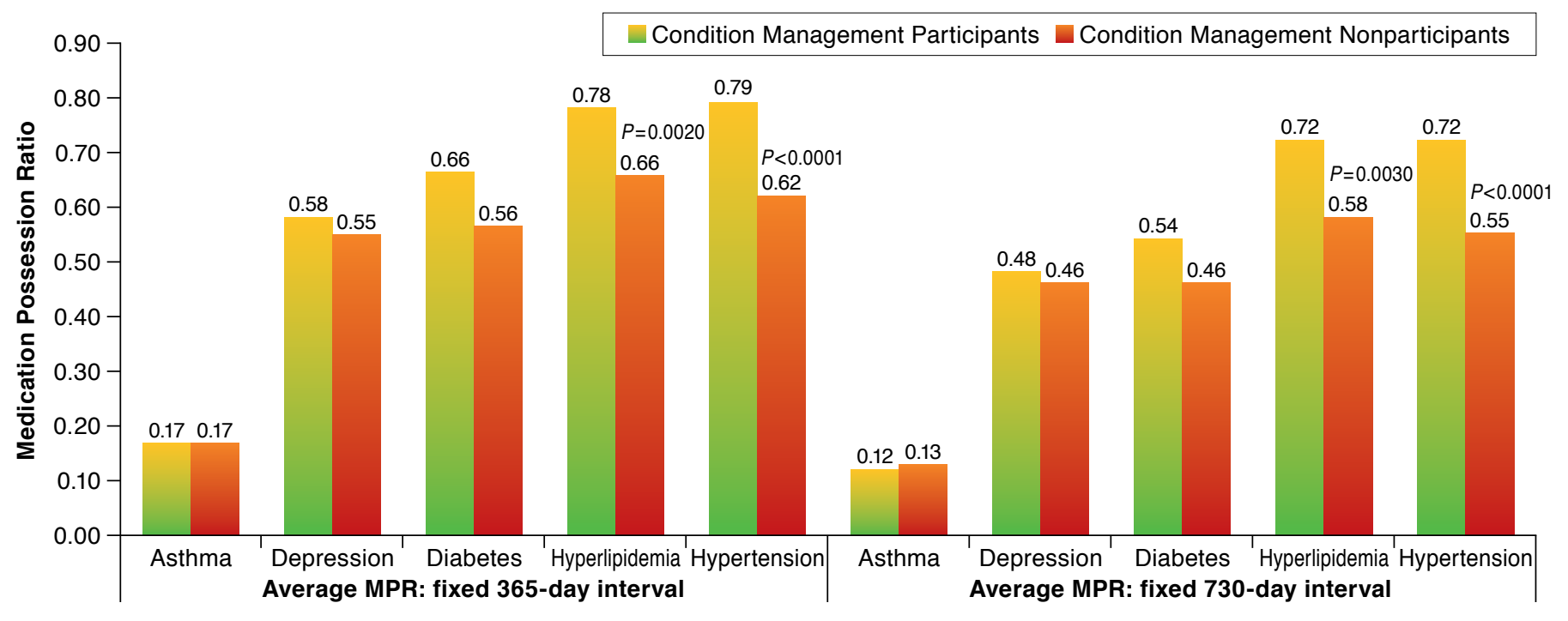

$M P R=$ medication possession ratio

demonstrated that MPRs continued to be statistically higher among subscribers and dependents who used onsite pharmacies, compared with those who used offsite pharmacies at 2 years $(P<0.001)$.

Medication persistence is another approach to evaluating the impact of onsite health services. Patwardhan et al. (2011) reported that 30 -day gaps in medication were $22 \%$ less frequent among workplace pharmacy users than those who used mailorder prescription services. ${ }^{30}$ Similarly, our analysis indicated that onsite pharmacy users were less likely to have a 30-day gap in coverage at 180 and 365 days. While this trend was not statistically significant across all medication types, the percentage of members without gaps was higher for almost all categories at both time points for members using onsite pharmacies.

Based on the results of our evidence review, this is the first study of onsite pharmacies to report the average time until treatment discontinuation. According our analysis, the average number of days until discontinuation was significantly longer $(P<0.0001)$ across medication types among onsite pharmacy users in our study. This measure is meaningful to consider, since onsite pharmacies may facilitate long-term medication persistence by offering members a more convenient and lower cost method of filling prescriptions.

The benefit of the onsite pharmacy on medication adherence was further demonstrated by the mixed model approach, which was used to assess the impact of the onsite pharmacy by adjusting for covariate effects. Compared with ordinary least square regression analysis, the mixed model approach not only allows adjustment of covariate effects but also has the advantage of controlling the potentially significant positive correlation effect if participants are simultaneously taking multiple types of medications. Considering all medication types together, members who used the onsite pharmacy were 3.44 times more likely to be adherent than those who used community pharmacies (95\% CI $=2.84-4.16 ; P<0.0001)$. Higher adherence was positively associated with age and CCI score.

Based on the results of the mixed model analysis, the adjusted medication adherence also tended to be higher among subscribers compared with dependents, but the difference was not significant at the 5\% level. This trend may suggest that convenient onsite health services have a slightly greater influence on subscribers because they are employees. However, there may be a spillover effect because dependents may also have regular access to onsite services. That is, in addition to the dependents who are also Cerner employees, other dependents may regularly visit the worksite for other reasons; for example, to attend clinic visits at the onsite health center or use the onsite fitness center. Some dependents under the age of 6 may also be enrolled at the onsite child care center.

In contrast, the mixed model analysis demonstrated a negative correlation with condition management participation. Several factors that were unaccounted for in the analysis may have influenced this finding. For example, condition management participants may have been more likely to subscribe to mail-order prescription services. Alternatively, the condition management programs may have been particularly effective and enabled some participants to successfully overcome their condition and thus eliminated the need for medication. Further studies investigating this trend are warranted. 
Based on the ordinary least squares regression analyses, across medication categories, MPR at 365 and 730 days was greater among members who participated in condition management programs. While not all of these findings were statistically significant, our results suggest a benefit of these services. Similar findings have been reported by other studies of employer-sponsored disease management programs. For example, Moore et al. (2013) reported MPR increases of approximately $2 \%$ following a hypertension and dyslipidemia medication therapy management program sponsored by an employer group. ${ }^{31}$

Onsite health services, especially employer-sponsored pharmacies, may reduce medication adherence barriers. Gellad et al. (2009) conducted a systematic literature review to catalog contributing factors to nonadherence. ${ }^{32}$ The authors noted that cost sharing and regimen complexity had previously been extensively researched and were known to influence adherence. However, Gellad et al. identified a number of other barriers commonly referenced across publications, including medication beliefs (i.e., perceived efficacy and tolerability), social support, and patient-provider relationship. Onsite pharmacies and condition management programs may improve medication adherence by providing members with a convenient and low-cost way to interact with providers and receive counseling to reduce adherence challenges. For example, the onsite pharmacy in this study was located at the worksite, offered medications at a reduced price, and provided automatic refills and refill reminders.

Future research should explore the association between employer-sponsored health services, including onsite pharmacies, with health care outcome, utilization, and costs. While medication adherence is an important endpoint, a correlation with health outcomes would provide additional evidence to justify investment in an onsite health center. Demonstration of positive health outcomes, improved productivity, and cost savings are particularly important to employers and other organizations managing the health services of a population. ${ }^{16,33}$

\section{Limitations}

Given the observational nature of this research, the conclusions of this study are limited by certain factors that could not be fully controlled. In particular, there may be underlying differences between members who choose to fill prescriptions onsite and those who use community pharmacies. Members who use the onsite pharmacy may be more likely to practice healthy lifestyles, including medication adherence. ${ }^{34,35}$ Additionally, the onsite pharmacy was used by employees more than their dependents, who are less likely to be at the worksite on a regular basis.

Other potential biases may influence onsite and offsite pharmacy users similarly. For example, discontinuation of treatment may indicate a favorable change in a member's condition. Likewise, both study groups included members with multiple conditions and depression, which are characteristics associated with lower medication adherence. ${ }^{36}$ Some metrics of medication adherence may not have been appropriate for all medication types. For example, rescue inhalers may not need to be refilled every 30 days, and inclusion of these therapies could have confounded measures of adherence-such as MPR - that assume a 30-day refill interval. Lastly, the results of this study may not be generalizable to other populations given that only members of 1 health plan were included.

The analysis of condition management participation was limited by the amount of available data. In particular, it was not possible to stratify the results by condition because the medication types included in the study (i.e., asthma, depression, diabetes, hyperlipidemia, and hypertension) were not directly aligned with the focus of the condition management programs (i.e., hypertension, diabetes, weight management, and depression).

\section{Conclusions}

Across multiple measures of medication adherence, the results of this study demonstrate a favorable association between the use of employer-sponsored health services, particularly onsite pharmacies, and medication adherence. While inconsistent findings were observed for the impact on condition management participation on adherence, compared with members who used offsite pharmacies, those who filled prescriptions onsite had higher medication adherence rates as measured even at the 2-year follow-up time point. Moreover, onsite pharmacy users exhibited better medication persistence and were less likely to discontinue treatment.

\section{Authors}

KATHLEEN M. AGUILAR, MPH, is Scientist, Cerner Research Consulting, Culver City, California; QINGJIANG HOU, MS, is Scientist, Cerner Quantitative Research, Kansas City, Missouri; and ROSS M. MILLER, MD, MPH, is Medical Director, Cerner Employer Services, Culver City, California.

AUTHOR CORRESPONDENCE: Kathleen M. Aguilar, MPH, Cerner Research Consulting, 600 Corporate Pointe, Ste. 320, Culver City, CA 90230. Tel.: 310.598.4462; E-mail: Kat.Aguilar@cerner.com.

\section{DISCLOSURES}

This research was conducted by Cerner Corporation, Kansas City, Missouri without external funding. All authors are employed by Cerner Corporation.

Study concept and design were contributed by Miller and Aguilar with assistance from Hou. Data were primarily collected by Hou, assisted by Aguilar and Miller, and interpreted by Hou, Aguilar, and Miller. The manuscript was primarily written by Aguilar, assisted by Hou and Miller, and revised by Aguilar, along with Miller, with assistance from Hou. 


\section{REFERENCES}

1. Cramer JA, Roy A, Burrell A, et al. Medication compliance and persistence: terminology and definitions. Value Health. 2008;11(1):44-47.

2. Simpson SH, Eurich DT, Majumdar SR, et al. A meta-analysis of the association between adherence to drug therapy and mortality. BMJ. 2006;333(7557):15.

3. Roebuck MC, Liberman JN, Gemmill-Toyama M, Brennan TA. Medication adherence leads to lower health care use and costs despite increased drug spending. Health Aff (Millwood). 2011;30(1):91-99.

4. Sokol MC, McGuigan KA, Verbrugge RR, Epstein RS. Impact of medication adherence on hospitalization risk and healthcare cost. Med Care. 2005;43(6):521-30

5. DiMatteo MR. Variations in patients' adherence to medical recommendations: a quantitative review of 50 years of research. Med Care. 2004:42(3):200-09

6. Gamble J, Stevenson M, McClean E, Heaney LG. The prevalence of nonadherence in difficult asthma. Am J Respir Crit Care Med. 2009;180(9):817-22.

7. Ho PM, Bryson CL, Rumsfeld JS. Medication adherence: its importance in cardiovascular outcomes. Circulation. 2009;119(23):3028-35.

8. Fischer MA, Stedman MR, Lii J, et al. Primary medication non-adherence: analysis of 195,930 electronic prescriptions. J Gen Intern Med. 2010;25(4):284-90.

9. Krousel-Wood M, Islam T, Webber LS, Re RN, Morisky DE, Muntner P. New medication adherence scale versus pharmacy fill rates in seniors with hypertension. Am J Manag Care. 2009;15(1):59-66.

10. Briesacher BA, Andrade SE, Fouayzi H, Chan KA. Comparison of drug adherence rates among patients with seven different medical conditions. Pharmacotherapy. 2008;28(4):437-43.

11. Yeaw J, Benner JS, Walt JG, Sian S, Smith DB. Comparing adherence and persistence across 6 chronic medication classes. J Manag Care Pharm. 2009;15(9):728-40. Available at: http://www.amcp.org/data/jmcp/728-740.pdf.

12. New England Healthcare Institute. Thinking outside the pillbox: a system-wide approach to improving patient medication adherence for chronic disease. August 2009. Available at: http://www.nehi.net/writable/publication_files/file/pa_issue_brief_final.pdf. Accessed June 18, 2015.

13. Osterberg L, Blaschke T. Adherence to medication. N Engl J Med. 2005;353(5):487-97.

14. Dillon MJ. Employer-sponsored health care services. J Manag Care Pharm. 2001;7(3):189-92. Available at: http://www.amcp.org/WorkArea/ DownloadAsset.aspx?id=6377.

15. Tao XG, Fagan PJ, LeNoach E, Hawkins M, Ross-Gavin M, Bernacki EJ. The relationship between a worksite wellness clinic and hospital emergency department visits. J Occup Environ Med. 2014;56(12):1313-18.

16. Baicker K, Cutler D, Song Z. Workplace wellness programs can generate savings. Health Aff (Millwood). 2010;29(2):304-11.

17. Naydeck BL, Pearson JA, Ozminkowski RJ, Day BT, Goetzel RZ. The impact of the highmark employee wellness programs on 4-year healthcare costs. J Occup Environ Med. 2008;50(2):146-56.

18. Kaspin LC, Gorman KM, Miller RM. Systematic review of employersponsored wellness strategies and their economic and health-related outcomes. Popul Health Manag. 2013;16(1):14-21.
19. Milani RV, Lavie CJ. Impact of worksite wellness intervention on cardiac risk factors and one-year health care costs. Am J Cardiol. 2009;104(10):1389-92. 20. Berry LL, Mirabito AM, Baun WB. What's the hard return on employee wellness programs? Harv Bus Rev. 2010;88(12):104-12, 142.

21. Munir F, Randall R, Yarker J, Nielsen K. The influence of employer support on employee management of chronic health conditions at work. J Occup Rehabil. 2009;19(4):333-44

22. Xu A, Chomutare T, lyengar S. Persuasive attributes of medication adherence interventions for older adults: a systematic review. Technol Health Care. 2014;22(2):189-98.

23. Zeber JE, Manias E, Williams AF, et al. A systematic literature review of psychosocial and behavioral factors associated with initial medication adherence: a report of the ISPOR medication adherence \& persistence special interest group. Value Health. 2013;16(5):891-900.

24. van Servellen G, Heise BA, Ellis R. Factors associated with antidepressant medication adherence and adherence-enhancement programmes: a systematic literature review. Ment Health Fam Med. 2011;8(4):255-71.

25. Petry NM, Rash CJ, Byrne S, Ashraf S, White WB. Financial reinforcers for improving medication adherence: findings from a meta-analysis. Am J Med. 2012;125(9):888-96.

26. DeFulio A, Silverman K. The use of incentives to reinforce medication adherence. Prev Med. 2012;55(Suppl):S86-S94.

27. Romano PS, Roost LL, Jollis JG. Further evidence concerning the use of a clinical comorbidity index with ICD-9-CM administrative data. J Clin Epi. 1993;46(10):1085-90

28. Wetmore JB, Mahnken JD, Rigler SK, et al. Association of race with cumulative exposure to statins in dialysis. Am J Nephrol. 2012;36(1):90-96.

29. Sherman BW, Frazee SG, Fabius RJ, Broome RA, Manfred JR, Davis JC. Impact of workplace health services on adherence to chronic medications. Am J Manag Care. 2009;15(7):e53-e59.

30. Patwardhan A, Davis J, Murphy P, Khandelwal N, Sherman B, Manfred J. The impact of 90-day prescriptions on adherence at workplace pharmacies compared to traditional mail order. Popu Health Manag. 2011;14(6):285-91.

31. Moore JM, Shartle D, Faudskar L, Matlin OS, Brennan TA. Impact of a patient-centered pharmacy program and intervention in a high-risk group. J Manag Care Pharm. 2013;19(3):228-36. Available at: http://www.amcp.org/ JMCP/2013/April/16321/1033.html.

32. Gellad WF, Grenard J, McGlynn EA. A review of barriers to medication adherence: a framework for driving policy options. Rand Health. 2009. Available at: http://www.rand.org/content/dam/rand/pubs/technical_reports/2009/RAND_TR765.pdf. Accessed June 18, 2015.

33. Goetzel RZ, Ozminkowski RJ. The health and cost benefits of work site health-promotion programs. Annu Rev Public Health. 2008;29:303-23.

34. Ladova K, Vlcek J, Vytrisalova M, Maly J. Healthy adherer effect - the pitfall in the interpretation of the effect of medication adherence on health outcomes. J Eval Clin Pract. 2014;20(2):111-16.

35. Molloy GJ, O'Carroll RE, Ferguson E. Conscientiousness and medication adherence: a meta-analysis. Ann Behav Med. 2014;47(1):92-101.

36. Grenard JL, Munjas BA, Adams JL, et al. Depression and medication adherence in the treatment of chronic diseases in the United States: a metaanalysis. J Gen Intern Med. 2011;26(10):1175-82. 